\title{
Análise das Estratégias Empregadas em um Programa de Intervenção Fonológica para Crianças com DeficiênCia Auditiva ${ }^{1,2}$ Analysis of Strategies Employed in a Phonological InterVention Program FOR HARD OF HEARING CHILDREN
}

\author{
Ana Luiza Cabral MARINHO3 \\ Bárbara Layse ROCHA E SILVA ${ }^{4}$ \\ Joseli Soares BRAZOROTTO5
}

\begin{abstract}
RESUMO: Programas de intervenção fonológica para crianças com deficiência auditiva têm sido estudados mundialmente, dados os seus comprovados benefícios para o desenvolvimento da leitura. No entanto, no Brasil, não há referência à avaliação desses programas para crianças com deficiência auditiva inseridas em serviços de (re)habilitação auditiva. O objetivo desta pesquisa foi analisar as estratégias empregadas em um programa de intervenção fonológica para crianças com deficiência auditiva. Trata-se de estudo do tipo exploratório, de fonte documental, longitudinal com abordagem qualitativa, realizado a partir da intervençấo com 11 crianças com deficiência auditiva e três pedagogas que acompanharam a intervenção em grupo. Foram analisados os 80 planos das sessóes e as 80 fichas de observação das pedagogas, além da descrição dos resultados do grupo focal realizado com as pedagogas ao término da intervenção. Observou-se a necessidade de adaptaçóes quanto às instruçóes, às estratégias e aos materiais utilizados, nessa ordem de frequência, assim como a utilizaçáo de estratégias de comunicaçáo e técnicas específicas para otimizar a compreensão e a execução das atividades pelas crianças. Concluiu-se que o programa de intervençáo fonológica para crianças com deficiência auditiva necessitou de adaptaçôes e destaca-se a importância da capacitação dos profissionais nos serviços de terapia especializada para essa atuaçáo.
\end{abstract}

PALAVRAS-CHAVE: Deficiências da audição. Terapia fonoaudiológica. Aprendizagem da leitura. Formação pedagógica. Modelos de Educação Especial.

ABSTRACT: Phonological intervention programs for hard of hearing children has been studied worldwide, given their proven benefits for literacy development. However, in Brazil, there is no reference to the evaluation of these programs for hearing impaired children inserted in auditory (re)habilitation services. The objective of this research was to analyze the strategies employed in a phonological intervention program for hard of hearing children. This is an exploratory study, from a documentary, longitudinal source with a qualitative approach, carried out from the intervention with 11 hearing impaired children and three educators who followed the group intervention. Eighty session plans and 80 observation sheets of the educators were analyzed, as well as the description of the results of the focus group carried out with the educators at the end of the intervention. It was observed the need for adaptations regarding the instructions, strategies and materials used, in this order of frequency, as well as the use of communication strategies and specific techniques to optimize children's understanding and execution of the activities. It was concluded that the phonological intervention program for children with hearing impairment needed adaptations and the importance of training professionals in specialized therapy services for this performance is highlighted.

KEYWORDS: Hearing impairment. Speech therapy. Learning to read. Pedagogical training. Models of Special Education.

\footnotetext{
${ }^{1}$ https://doi.org/10.1590/1980-54702020v26e0018

${ }^{2}$ Agradecemos ao Centro SUVAG pela constante parceria; às fonoaudiólogas Ana Caroline Esther, Carolina Karla de Souza Evangelista, Darling Dayenne Santana Freire Carvalho, Genielly Barbosa Tavares e Taise Ferreira de Lima, pelo envolvimento na elaboração das estratégias lúdicas e durante a coleta de dados; e à fonoaudióloga Ms. Gabriela Rabelo, pelas importantes contribuiçôes nas discussóes deste trabalho.

${ }^{3}$ Departamento de Fonoaudiologia da Universidade Federal do Rio Grande do Norte. Natal/RN/Brasil. E-mail: aluiza.marinho@ gmail.com. ORCID: https://orcid.org/0000-0003-4415-870X

${ }^{4}$ Departamento de Fonoaudiologia, Universidade Federal do Rio Grande do Norte. Natal/RN/Brasil. Centro SUVAG do RN, Natal/RN, Brasil. E-mail: barbara_layse@hotmail.com. ORCID: https://orcid.org/0000-0002-9275-9326

${ }^{5}$ Departamento de Fonoaudiologia e Pós-Graduação em Gestão e Inovação Tecnológica em Saúde, Universidade Federal do Rio Grande do Norte. Laboratório de Inovação Tecnológica em Saúde do Hospital Universitário Onofre Lopes, Universidade Federal do Rio Grande do Norte (LAIS/HUOL/UFRN). Natal/RN/Brasil. E-mail: brazorotto@yahoo.com. ORCID: https://orcid. org/0000-0002-3891-9819
} 


\section{Introduçấo}

Nos últimos 30 anos, avanços tecnológicos e científicos surpreendentes têm proporcionado às crianças com deficiência auditiva o acesso aos sons da fala no momento favorável ao seu desenvolvimento da linguagem (Wake \& Carew, 2016; Bornstein, 2018). Ainda assim, devido às variáveis intervenientes ao processo de (re)habilitação - tais como: a idade no diagnóstico, causa e grau da deficiência auditiva, o tempo de uso dos dispositivos auxiliares à audição, o envolvimento das famílias no processo terapêutico (Bicas, Guijo, \& Delgado-Pinheiro, 2017; Moret, 2017; Oliveira, Penna, \& Lemos, 2015; Penna, Lemos, \& Alves, 2015; Tabaquim et al., 2013; Vasconcelos \& Pereira, 2015), entre outros fatores -, crianças com deficiência auditiva apresentam desvantagem em relação às crianças ouvintes quanto ao processamento fonológico e são, portanto, uma população de risco para a aquisição da leitura (Goldberg \& Lederberg, 2015; Lederberg, Schick, \& Spencer, 2013; Naveka von Mentzer et al., 2013; Werfel, Douglas, \& Ackal, 2016).

Destaca-se que, para as crianças com deficiência auditiva, a apreensão do código escrito é especialmente importante pois essa aquisição fortalece o domínio da linguagem oral, além do desenvolvimento apropriado de habilidades cognitivas essenciais para muitas de suas realizações (Gabriel, Morais, \& Kolinsky, 2016; Lemes \& Goldfeld, 2008).

Considera-se que o trabalho de desenvolvimento das habilidades auditivas realizado com essas crianças nos programas de (re)habilitação auditiva, associado ao treino das habilidades metalinguísticas, em especial a consciência fonológica, são os protagonistas para um bom desempenho linguístico (Gamba-Yoshida, 2017; Goldberg \& Lederberg, 2015; Spencer, Schuele, Guillot, \& Lee, 2008). De acordo com os referidos autores, a consciência fonológica é a capacidade de analisar e manipular os sons de uma língua.

Quanto às abordagens terapêuticas para as crianças com deficiência auditiva, a Abordagem Aurioral é amplamente utilizada no Brasil, quando as famílias escolhem o caminho do desenvolvimento auditivo para a aquisição da linguagem oral de seus filhos (Bevilacqua $\&$ Formigoni, 2012). Tal abordagem prioriza a via sensorial auditiva em situações interacionais de linguagem como meta para o desenvolvimento da linguagem oral, respondendo às necessidades psicológicas, sociais e educacionais das crianças com deficiência auditiva e de seus familiares (Moret, 2017).

Para tanto, as crianças (re)habilitadas por meio dessa abordagem frequentam, comumente, terapias fonoaudiológicas individuais, com a participação dos familiares e, habitualmente, os serviços especializados de (re)habilitação oferecem também atendimentos fonoaudiológicos e/ou pedagógicos em enquadre de grupo, considerando os aspectos positivos da motivação do trabalho em pequenos grupos com as crianças, além do custo terapêutico, de modo a otimizar tanto a quantidade de atendimentos prestados quanto a qualidade da estimulação oferecida (Bevilacqua \& Formigoni, 2012; Moret, 2017).

Nos atendimentos individuais e grupais, uma série de estratégias de comunicação e de técnicas para capitalizar a capacidade auditiva das crianças com os dispositivos eletrônicos utilizados por elas, a saber, em especial, os Aparelhos de Amplificação Sonora Individual (AASI) e/ou Implantes Cocleares (IC), é empregada pelos terapeutas, de modo a favorecer a 
comunicação da forma mais fluida possível para que a criança perceba-se como um comunicador competente (Granha, Oliveira, \& Rumpf, 2008; Resegue-Coppi, 2008).

Com base na premissa de que todas as crianças com deficiência auditiva, se bem adaptadas aos recursos auxiliares à audição e envolvidas em um processo terapêutico adequado, deverão acessar o código linguístico apropriadamente, o desenvolvimento da linguagem escrita nesta população fundamenta-se nas habilidades requeridas para as crianças ouvintes (Bergeron, Lederberg, Easterbrooks, Miller, \& Connor, 2009; Easterbrooks, Lederberg, \& Connor, 2010; Harris, Terlektsi, \& Kyle, 2017; Lederberg, Miller, Easterbrooks, \& Connor, 2014; Mahapatra, 2015).

Easterbrooks, Lederberg e Connor (2010), em um estudo descritivo, observaram salas de ensino infantil e fundamental. Os pesquisadores utilizaram as informaçóes baseadas no protocolo de observação para desenvolver um currículo inicial de alfabetização denominado Foundations for Literacy. A premissa dos referidos autores era a de que é possível alfabetizar crianças com deficiência auditiva utilizar os mesmos construtos da alfabetização para as crianças ouvintes, desde que fossem realizadas adaptaçóes que apoiassem as crianças com deficiência auditiva. O currículo elaborado focalizou-se no ensino de consciência fonológica, fonética, fluência, vocabulário e linguagem com adaptaçóes adequadas. Seus resultados após a aplicação com crianças com deficiência auditiva demonstraram que elas poderiam aprender correspondências fonema-grafema específicas por meio de instrução de habilidades auditivas explícitas com suporte de linguagem e apoio visual.

Em um estudo de abordagem experimental controlada com 37 pré-escolares com deficiência auditiva, Gilliver, Cupples, Ching, Leigh e Gunnourie (2016) constataram que o treino específico de consciência fonológica foi superior ao treino de vocabulário do grupo controle, e, que este tipo de intervenção é, portanto, altamente benéfico para crianças com deficiência auditiva. Resultados semelhantes confirmam que o treino específico da consciência fonológica é positivo para as crianças com deficiência auditiva em idades pré e escolar, inclusive os treinos computadorizados ou que utilizam recursos da teleprática (Chen, 2014; Lee, Hall, \& Sancibrian, 2017; Miller, Lederberg, \& Easterbrooks, 2013; Nakeva von Mentzer et al., 2013; Rakhshanfadaee \& Salehi, 2016; Wang, Spychala, Harris, \& Oetting, 2013; Werfel, Douglas, \& Ackal, 2016).

Em nosso país, a escassez de estudos sobre a intervenção fonológica com a população de crianças com deficiência auditiva traz à tona a necessidade de se construir ou adaptar programas específicos de instrução dessas habilidades, os quais buscam desenvolver, de modo geral: a relação grafema-fonema, a rima, a aliteração, a manipulação silábica, a manipulação fonêmica, a identificação de palavras em frases (Capellini et al., 2010), a memória de trabalho, incluindo também a leitura e a escrita em etapas posteriores (Salgado, 2010). É importante, pois, que se investigue a melhor maneira de se trabalhar tais habilidades com as crianças deficiência auditiva, já que, certamente, adaptações são necessárias, conforme referido pela literatura (Easterbrooks, Lederberg, \& Connor, 2010; Lederberg et al., 2014; Lee, Hall, \& Sancibrian, 2017).

Além disso, a instrução aos professores ou pedagogos para o trabalho de treino específico com a consciência fonológica é fundamental (Capellini et al., 2010; Messier \& Jackson, 2014; Spencer, Schuele, Kathryn, \& Marvin, 2008) e, portanto, nos programas de (re)habili- 
tação auditiva é imprescindível que a equipe de terapeutas possa receber a formação adequada para o trabalho específico de intervenção fonológica. Nesse sentido, o objetivo deste estudo foi analisar as estratégias de um programa de intervenção fonológica aplicado em grupo para crianças com deficiência auditiva, de acordo com a avaliação dos pesquisadores e dos pedagogos, terapeutas dessas crianças.

\section{Materiais e MÉTOdos}

Trata-se de um estudo do tipo exploratório, de fonte documental, longitudinal com abordagem qualitativa, realizado no Centro SUVAG RN, um serviço de saúde auditiva de alta complexidade do Sistema Único de Saúde, de natureza filantrópica. É importante esclarecer que este estudo é parte de uma pesquisa mais ampla que buscou analisar a efetividade do programa de intervenção fonológica elaborado para o trabalho em grupo para crianças com deficiência auditiva, cuja execução foi aprovada pelo Comitê de Ética em Pesquisa (CEP) da Universidade Federal do Rio Grande do Norte (UFRN), sob Parecer de número 1.144.295.

Para a pesquisa da efetividade da intervenção fonológica, as crianças foram divididas, de modo aleatório, em grupos controle (11 crianças) e experimental (11 crianças), sendo constatada a mudança clínica positiva estatisticamente significativa do grupo experimental em relação ao grupo controle, nas avaliações pós-intervenção. Salienta-se que o presente estudo enfatizou a análise qualitativa do programa, com foco nas estratégias empregadas e não na análise estatística pré e pós-intervenção. A amostra foi selecionada por conveniência, sendo composta pelas três pedagogas atuantes no setor de (re)habilitação auditiva e por 11 crianças com deficiência auditiva sensorioneural de graus moderado a profundo, usuárias de AASI, na faixa etária de 8 a 14 anos. Os pedagogos participantes da pesquisa bem como as famílias das crianças com deficiência auditiva foram esclarecidos sobre o estudo e todos aceitaram participar dele e assinaram, assim, o Termo de Consentimento Livre e Esclarecido (TCLE).

No início de cada sessão, as pesquisadoras entregavam uma ficha de observação às pedagogas responsáveis por cada turma. Nessa ficha, elas fizeram anotaçóes sobre a sessão de acordo com o desempenho de cada criança e o desempenho geral da turma, diante das estratégias realizadas. Essas fichas de observação serão também analisadas nesta pesquisa.

Todas as crianças mencionadas frequentavam terapias fonoaudiológicas e pedagógicas em enquadres individuais e em grupo, tendo como meta a (re)habilitação auditiva. Tais atendimentos eram distribuídos de duas a três vezes por semana, com duração de 50 minutos, em um período do dia - no contraturno, pois as crianças frequentavam escola regular (Ensino Fundamental I).

O trabalho de intervenção ocorreu dentro da rotina de atendimentos das 11 crianças do grupo experimental, sendo, portanto, acompanhado pelas pedagogas do serviço. Seis pesquisadoras participaram do estudo. Uma escala foi organizada, fazendo com que cada duas pesquisadoras fossem responsáveis pela intervenção em um dos grupos: uma dirigia a sessão, e a outra funcionava como apoio, realizava as anotaçóes dos resultados, organizava os materiais, etc. Todas as seis pesquisadoras receberam treinamento para a aplicação das estratégias e todas participaram da elaboração das 200 atividades destinadas ao trabalho de intervenção 
fonológica. Vale ressaltar que uma habilidade poderia ser trabalhada por meio de mais de uma estratégia, por sessão.

Os grupos das crianças eram divididos de acordo com a sua faixa etária, desenvolvimento das habilidades auditivas e da linguagem oral, da leitura e da escrita, critérios estabelecidos pelo serviço de (re)habilitação auditiva. Tais grupos eram identificados por cores, a saber: roxo, azul, laranja e marrom, os quais estão apresentados na Tabela 1 mais adiante.

A intervenção fonológica foi baseada no modelo de remediação de Capellini et al. (2010), Salgado (2010) e na revisão da literatura sobre programas de intervenção fonológica para crianças com deficiência auditiva em outras línguas (Lederberg et al., 2014; Lee, Hall, \& Sancibrian, 2017; Spencer et al., 2008). Cada sessão contou com uma média de quatro crianças por turma. Foi previamente elaborado um arquivo com a descrição de todas as estratégias, por habilidade, que foram trabalhadas ao decorrer do estudo, totalizando 200 estratégias. Foram elencadas oito habilidades para serem trabalhadas em todas as 20 sessóes, sendo estas: 1) Correspondência grafema-fonema; 2) Discriminação de fonemas em sílabas; 3) Rima; 4) Classificação de palavras pares; 5) Adição, subtração e manipulação silábica e fonêmica; 6) Aliteração; 7) Correspondência fonema-grafema; e 8) Memória de Trabalho Fonológica.

Todas as 80 sessóes, no total de 20 sessóes para cada um dos 4 grupos, foram descritas em plano diário de terapia, os quais serão analisados neste estudo. Desse modo, o material de análise para esta pesquisa constou de 80 planos diários preenchidos com as avaliaçóes das pesquisadoras e de 80 fichas de observação preenchidas pelas pedagogas, totalizando 160 formulários. Além disso, para a análise geral da percepção das pedagogas sobre o programa de intervenção fonológica, foi realizado um grupo focal (Trad, 2009) após a finalização da intervenção.

\section{Resultados}

Nesta seção, serão apresentadas as análises descritivas dos 160 formulários, inclusos os planos diários de cada sessão de intervenção, bem como os formulários de observação das pedagogas, além da avaliação delas sobre o programa de intervenção, coletada por meio do grupo focal. Para aproximar a caracterização das crianças participantes do estudo dos resultados mencionados, exibe-se na Tabela 1, a seguir, as características dessas crianças.

\begin{tabular}{|c|c|c|c|c|c|c|c|c|c|c|c|}
\hline $\begin{array}{l}\text { CARACTERÍ́STICAS } \\
\text { DAS CRIANÇAS }\end{array}$ & $\mathrm{C} 1$ & $\mathrm{C} 2$ & C3 & $\mathrm{C} 4$ & C5 & $\mathrm{C} 6$ & C7 & C8 & C9 & $\mathrm{C} 10$ & C11 \\
\hline Grau PA - OD & M & S & M & S & M & M & S & S & M & S & M \\
\hline Grau PA - OE & - & $S$ & M & $S$ & $S$ & M & S & $S$ & $S$ & S & M \\
\hline Idade & $\begin{array}{c}8 \mathrm{a} \\
11 \mathrm{~m}\end{array}$ & $9 \mathrm{a} 6 \mathrm{~m}$ & $\begin{array}{l}10 \mathrm{a} \\
11 \mathrm{~m}\end{array}$ & 11 a $1 \mathrm{~m}$ & $\begin{array}{c}11 \mathrm{a} \\
5 \mathrm{~m}\end{array}$ & $\begin{array}{l}11 \mathrm{a} \\
2 \mathrm{~m}\end{array}$ & $\begin{array}{l}12 \mathrm{a} \\
7 \mathrm{~m}\end{array}$ & $\begin{array}{l}13 \mathrm{a} \\
3 \mathrm{~m}\end{array}$ & $\begin{array}{c}13 a \\
4 m\end{array}$ & $\begin{array}{l}12 \mathrm{a} \\
5 \mathrm{~m}\end{array}$ & $\begin{array}{l}14 \mathrm{a} \\
7 \mathrm{~m}\end{array}$ \\
\hline TPS & $\begin{array}{c}7 \mathrm{a} \\
11 \mathrm{~m}\end{array}$ & 5 a $7 m$ & $\begin{array}{c}8 \mathrm{a} \\
11 \mathrm{~m}\end{array}$ & $1 \mathrm{a} 2 \mathrm{~m}$ & $7 \mathrm{a}$ & 6 a $3 m$ & 3 a $9 \mathrm{~m}$ & $\begin{array}{c}7 \mathrm{a} \\
10 \mathrm{~m}\end{array}$ & 7 a $4 \mathrm{~m}$ & $5 \mathrm{a} 0 \mathrm{~m}$ & 9 a $6 \mathrm{~m}$ \\
\hline Idade Auditiva & 1a & $\begin{array}{c}3 \mathrm{a} \\
11 \mathrm{~m}\end{array}$ & $\begin{array}{c}2 \mathrm{a} \\
11 \mathrm{~m}\end{array}$ & 9 a $11 \mathrm{~m}$ & 4 a $5 m$ & $\begin{array}{c}4 \mathrm{a} \\
11 \mathrm{~m}\end{array}$ & $\begin{array}{c}8 \mathrm{a} \\
10 \mathrm{~m}\end{array}$ & $\begin{array}{l}5 \mathrm{a} \\
7 \mathrm{~m}\end{array}$ & $6 a$ & 7 a $5 m$ & $\begin{array}{c}4 \mathrm{a} \\
11 \mathrm{~m}\end{array}$ \\
\hline Dispositivo & AASI & AASI & AASI & AASI & AASI & AASI & AASI & AASI & AASI & AASI & AASI \\
\hline
\end{tabular}




\begin{tabular}{|c|c|c|c|c|c|c|c|c|c|c|c|}
\hline $\begin{array}{l}\text { CARACTERÍSTICAS } \\
\text { DAS CRIANÇAS }\end{array}$ & C1 & C2 & C3 & C4 & C5 & C6 & C7 & C8 & C9 & C10 & C11 \\
\hline $\begin{array}{l}\text { Tempo de uso dos } \\
\text { Dispositivos (h/ } \\
\text { dia) OD }\end{array}$ & 11 & 6 & 5 & 13 & 12 & 9 & 8 & 4 & 13 & 12 & 9 \\
\hline $\begin{array}{l}\text { Tempo de uso dos } \\
\text { Dispositivos (h/ } \\
\text { dia) OE }\end{array}$ & - & 6 & 5 & 13 & 12 & 5 & 9 & 3 & 13 & 12 & 9 \\
\hline $\begin{array}{l}\text { Participação da } \\
\text { Família na (Re) } \\
\text { habilitação (EEF) }\end{array}$ & 4 & 4 & 4 & 3 & 3 & 3 & 2 & 4 & 3 & 3 & 4 \\
\hline $\begin{array}{l}\text { Reside na cidade } \\
\text { do Serviço de }(\mathrm{Re}) \\
\text { habilitação }(\mathrm{S} / \mathrm{N})\end{array}$ & $\mathrm{N}$ & $S$ & $\mathrm{~S}$ & $S$ & $S$ & $S$ & $\mathrm{~N}$ & $\mathrm{~N}$ & $S$ & $S$ & $S$ \\
\hline $\begin{array}{l}\text { Categoria de } \\
\text { Audiçấo }\end{array}$ & 6 & 5 & 6 & 5 & 5 & 3 & 5 & 6 & 5 & 6 & 5 \\
\hline $\begin{array}{l}\text { Categoria de } \\
\text { Linguagem }\end{array}$ & 5 & 3 & 5 & 4 & 5 & 5 & 4 & 5 & 4 & 5 & 4 \\
\hline $\begin{array}{l}\text { Usa Sistema de } \\
\text { FM }(\mathrm{S} / \mathrm{N})\end{array}$ & $\mathrm{N}$ & $S$ & $S$ & $S$ & $\mathrm{~N}$ & $S$ & $\mathrm{~N}$ & $S$ & $S$ & $S$ & $S$ \\
\hline $\begin{array}{l}\text { Idade de Ingresso } \\
\text { na (Re)Habilita- } \\
\text { çấo (anos) }\end{array}$ & 7 & 6 & 6 & 1 & 7 & 7 & 4 & 8 & 8 & 6 & 13 \\
\hline
\end{tabular}

Tabela 1: Caracterização das crianças participantes e sua organização nos grupos de atendimento. Fonte: Elaborada pelas autoras, Natal/RN, 2018

Legenda: PA - OD: Perda auditiva da orelha direita; PA - OE: Perda auditiva da orelha esquerda; TPS: Tempo de Privação Sensorial: tempo que a criança ficou privada de exposição aos sons, anterior à adaptação dos dispositivos eletrônicos auxiliares à audição; Idade Auditiva: Tempo que a criança usou os dispositivos auxiliares à audição (neste caso, o AASI), desde que foi adaptada; AASI: Aparelho de Amplificação Sonora Individual; Bilat: Bilateral; EEF: Escala de Envolvimento Familiar: escala de análise da participação familiar com notas de 1 a 5, sendo 1 participação inferior à necessária e 5 participação ótima; S: Sim; N: Não; Categoria de Audição: categorização da criança de acordo com seu desempenho auditivo, com escores de 0 a 6 , sendo 0 - náo detecta a fala até 6 - compreende o discurso oral, sem uso de leitura orofacial; Categoria de Linguagem: análoga à categoria de audição, trata-se da categorização da criança de acordo com seu desempenho linguístico oral, sendo 0 - não usa fala até 5 - usa discurso oral complexo; Sistema de FM: Sistema de Frequência Modulada - dispositivo auxiliar à audição utilizado em geral no ambiente escolar, que permite à criança captar a fala do professor sem a interferência da distância, minimizando os efeitos do ruído e da reverberação da sala de aula, com benefícios para o acesso ao conteúdo em sala de aula.

Observou-se, na caracterização das crianças participantes do estudo, que se trata de crianças com perdas auditivas sensorioneurais de graus moderado e severo, com média de tempo de privação sensorial de 7 anos e 9 meses e média de idade auditiva de 6 anos. Com exceção da C4, todas as demais crianças apresentavam tempos de privação sensorial auditiva (tempo em que estiveram sem equipamentos auxiliares à audição) superiores aos recomendados e idade auditiva (tempo de uso dos dispositivos desde sua adaptação) que justificam a necessidade de 
intervenções focalizadas em audição, linguagem oral e habilidades metalinguísticas, mesmo em escolares desta faixa etária (média da amostra - 11 anos).

Das 11 crianças, 10 eram usuárias de aparelhos auditivos bilateralmente, e uma criança utilizava o AASI na orelha direita. A média de uso diário dos aparelhos auditivos era de nove horas, indicando uso regular dos dispositivos, mas ainda aquém do desejável (o tempo ideal deve ser de 14 a 16 horas/dia). Sobre seu desempenho auditivo, excetuando a C6, todas as demais se apresentaram nas categorias de audição 5 ou 6 , indicando que eram crianças que identificavam o significado da maior parte do que era dito a elas, sem a necessidade de leitura orofacial ou que compreendiam utilizando apenas a audição, respectivamente. Quanto ao desenvolvimento da linguagem oral, com exceção da C2, avaliada como produzindo frases curtas, as demais crianças apresentaram discurso oral, ainda que não em etapa madura (correspondente à categoria 4 de linguagem).

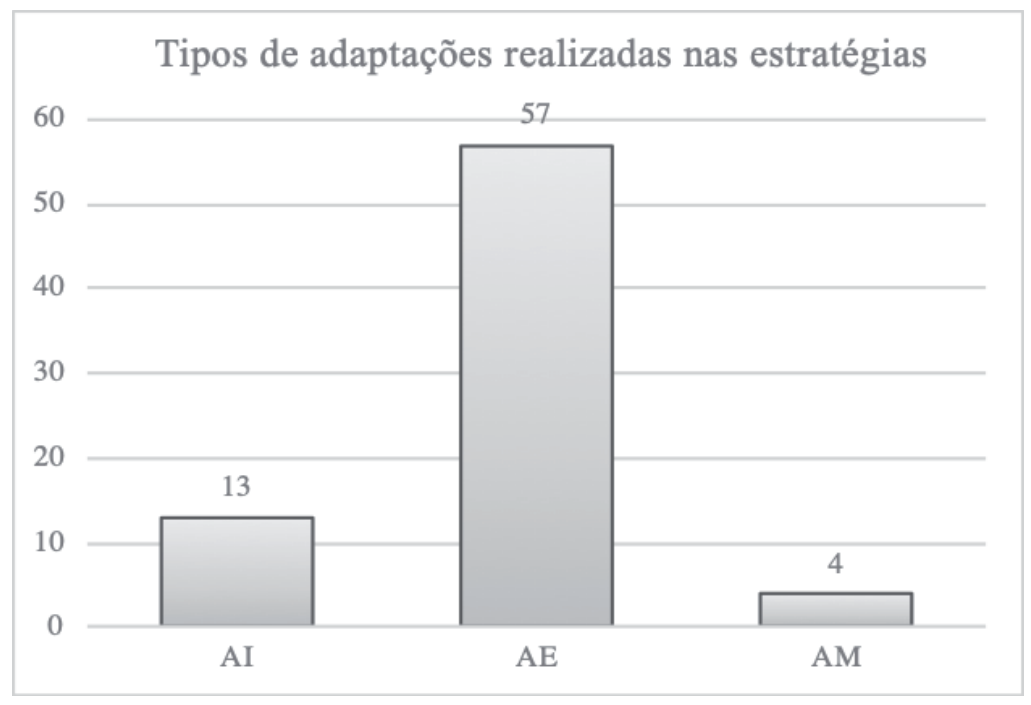

Figura 1: Quantidade dos tipos de adaptaçóes realizadas na intervenção fonológica em grupo. Fonte: Elaborada pelas autoras

Legenda: AI: Adaptação durante a instrução da estratégia; AE: Adaptação da estratégia em si; AM: Adaptação de materiais.

As adaptaçóes realizadas em cada uma das sessóes foram descritas; assim, observou-se, a partir da análise, que as adaptaçóes de instrução, ocorrentes em menor quantidade (13), referiram-se às dicas para ajudar as crianças a compreender a atividade, ao uso de objetos concretos durante a instrução para facilitar a sua compreensão, à repetição da instrução, ao uso de sinônimos e à diminuição da velocidade de fala, o que também foi observado durante a realização das atividades, por meio da análise dos planos diários de terapia em grupo, quanto ao uso das técnicas e das estratégias de comunicação pelas pesquisadoras.

Quanto às adaptações na execução das estratégias (57), destacaram-se a diminuição do número de itens de treino, em especial a memória de dígitos, ou o número de rodadas de cada habilidade a ser trabalhada, no caso das habilidades de manipulaçáo fonêmica, o uso de destaques acústicos, especialmente na atividade de discriminação de fonemas em sílabas, bem 
como o uso de leitura orofacial, além da necessidade de apoio visual (palavras ou figuras e objetos) que auxiliasse as crianças a realizar a manipulação silábica e fonêmica.

A adaptaçóes dos materiais (4) foram observadas em menor quantidade e referiram-se ao uso de palavras escritas ou figuras extras para apoiar as atividades. Observou-se, a partir da análise dos planos diários e das fichas de observação, que as habilidades em que as crianças exibiram maior dificuldade foram: a discriminação de fonemas em sílabas, a memória de dígitos e a consciência fonêmica, todas que exigem desempenho auditivo esperado para a faixa etária e escolaridade das crianças envolvidas neste estudo. Contudo, conforme observado na Tabela 1, essa amostra caracteriza-se por crianças mais velhas (8 a 14 anos), que já deveriam apresentar certo domínio das habilidades fonológicas. Como possíveis interferentes em seu desempenho, destacam-se os tempos de privação sensorial longos, o uso moderado ou mínimo dos AASIs por algumas das crianças e o ingresso tardio no programa de (re)habilitação.

$\mathrm{Na}$ análise dos planos diários por grupo, observou-se que o grupo marrom (GM) necessitou de adaptação de estratégias em todas as sessões realizadas em, no mínimo, uma habilidade, seguido do grupo azul (GA), grupo laranja (GL) e grupo roxo (GR), consecutivamente.

Sobre as técnicas utilizadas para otimizar a comunicação e a escuta das crianças nas situaçôes da intervenção, a partir da análise geral dos 80 planos diários, as pesquisadoras informaram, em ordem de frequência, que as técnicas mais utilizadas foram: destaques acústicos (consiste em dar ênfase a determinado som ou a determinada palavra dita) com 40\% de ocorrência nas 20 sessóes, seguida de apoio visual com 15\%, que consistiu no uso de objetos ou figuras, uso de repetição ou refraseamento, com 10\% e diminuição da velocidade de fala e ênfase nas palavras-chave, ambas as técnicas com 5\% de ocorrência. Observou-se, portanto, que o programa de intervenção fonológica delineado necessitou de adaptaçóes de várias naturezas, além do uso de estratégias de comunicação e de técnicas para melhorar a audibilidade para as crianças, de modo a adequar-se ao público-alvo.

Sobre a avaliação geral das pedagogas sobre o programa de intervenção, a Figura 2 apresenta o resultado das falas das 3 pedagogas. 


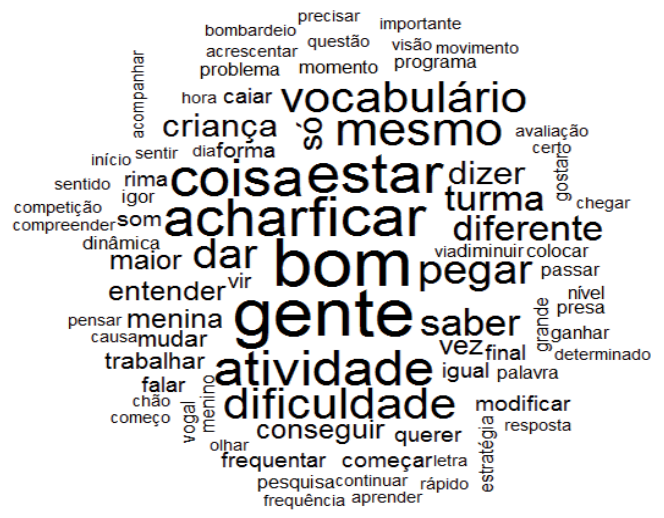

Figura 2: Análise da frequência de palavras a partir do roteiro do grupo focal. Fonte: Elaborada pelas autoras

A partir da análise da frequência de palavras pode-se observar que a avaliação das pedagogas sobre o programa de intervenção foi positiva (destacam-se as palavras bom e gente) e que elas se mostraram interessadas em continuar com a proposta (com destaque para a palavra ficar). Conforme notou-se em alguns dos comentários analisados, por sessão, as pedagogas destacaram que as atividades foram enriquecedoras para elas e que algumas foram utilizadas em sua prática clínica em outros grupos do serviço de (re)habilitação.

(S8): "As estratégias para a realização das atividades foram ótimas e bastante diversificadas."

(S11): "Gostei das estratégias para cada atividade, o que favoreceu o envolvimento do grupo bem como a melhor compreensáo por parte da maioria dos participantes."

(S 13): "Boas estratégias em cada atividade, excelente interação da terapeuta com o grupo e bons resultados."

Foi ainda referido que, nas primeiras sessóes, as crianças tiveram dificuldades, especialmente quanto à compreensão para participarem das atividades e quanto ao tempo da sessão para o trabalho com várias habilidades.

(S3): "Para a compreensão do grupo, acho as atividades rápidas, o tempo é curto para aplicar todas as atividades."

(S4): "As crianças se envolvem bem, ficam atentas às estratégias, mas demonstram dificuldades em entender como fazer a atividade."

(S6): "O tempo para cada atividade é pouco e eles precisam de mais tempo para compreender melhor o que é solicitado e treinar mais em cada sessão." 
Notou-se, entretanto, que, após a nona sessão, as pedagogas observaram tempos de resposta mais rápidos e melhor compreensão das crianças, mesmo nas atividades em que ainda apresentavam dificuldade em executar.

(S9): "As atividades elaboradas para as crianças foram bastante interessantes. Observei uma evolução de segunda-feira para hoje no sentido de melhor compreensão e melhores respostas."

(S11): "Todos compreenderam as instruções dada a cada atividade, as estratégias foram ótimas, o que facilitou o envolvimento de todos."

(S19): “Todos participaram com bastante interação e tiveram boa compreensão do que foi orientado."

Os aspectos destacados pelas pedagogas também puderam ser notados na análise dos planos diários das sessōes, em que, a partir da nona sessão, mesmo sem a alteração do tempo da sessão, as crianças passaram a executar com mais facilidade as atividades propostas, de acordo com os resultados descritos pelas pesquisadoras. Além disso, ainda foi observado, na análise dos planos diários, uma diminuição no uso de estratégias de comunicação e técnicas na metade do programa até o final das sessões. Esse fato pode ser atribuído à melhora das crianças na compreensão do trabalho fonológico, bem como ao aprimoramento das pesquisadoras como terapeutas desses grupos, o que envolve a relação pesquisador-criança e até mesmo a familiaridade das crianças com a fala de cada pesquisadora, fato também notado pelas pedagogas.

(S13): "Boas estratégias em cada atividade, excelente interaçấo da terapeuta com o grupo e bons resultados."

Quanto ao último questionamento do grupo focal, sobre as sugestóes para a melhoria do programa de intervenção fonológica, as educadoras destacaram: a necessidade do trabalho de intervenção com o vocabulário das crianças, visto que notaram o quanto o déficit apresentado por algumas das crianças teve impacto na compreensão da estratégia e no aproveitamento de todo o grupo no tempo previsto para cada sessão; a ampliaçáo do tempo da sessão ou a diminuição do número de atividades por sessão, para grupos com maior dificuldade; ou, ainda, o aumento do número de sessóes.

(S3): "Para a compreensão do grupo, acho as atividades rápidas, o tempo é curto para aplicar todas as atividades. Acredito que seja a dinâmica do trabalho o bombardeio de informaçóes."

(S8): "O tempo da atividade é muito pouco."

(S15): "Eles apresentam muita dificuldade na compreensão, no vocabulário e na memória, o que dificulta muito o trabalho."

O Quadro 1 a seguir apresenta as observaçóes das pedagogas sobre cada uma das sessóes de intervenção. 


\begin{tabular}{|c|c|}
\hline Sessão & Análise das Pedagogas \\
\hline 1 & $\begin{array}{l}\text { "O trabalho desenvolvido foi de consciência fonológica, no qual as crianças tinham que falar o nome das letras e dizer } \\
\text { o som de cada uma. Os quatro começaram timidamente. As crianças já tinham esse conhecimento, foi super fácil."; } \\
\text { "Atividades bem dinâmicas. As crianças se mantiveram interessadas o tempo todo e apresentaram dificuldade nos sons } \\
\text { dos fonemas. Reconheceram até mais letras do que eu esperava. Foi muito bom." }\end{array}$ \\
\hline 2 & $\begin{array}{l}\text { "Muito boas as atividades, eles interessados o tempo todo. A grande dificuldade é a memorizaçáo e a compreen- } \\
\text { são de ordens simples do que é para fazer."; "As crianças se mostraram tímidas inicialmente, mas depois foram se } \\
\text { soltando, porém demonstraram muita dificuldade em entender o desenvolvimento das atividades. Acho o tempo } \\
\text { muito curto, pois o grupo apresentou dificuldades na compreensão das atividades."; "Dificuldade quanto à } \\
\text { lembrança do som da letra, rimar algumas palavras. As crianças sentiram dificuldade devido à limitaçáo do voca- } \\
\text { bulário. No entanto a turma já tem conhecimento sobre rimas, palavras onde acrescentamos sílabas e retiramos } \\
\text { sílabas, nomes das letras e seu som..." }\end{array}$ \\
\hline 3 & $\begin{array}{l}\text { "Para a compreensão do grupo, acho as atividades rápidas, o tempo é curto para aplicar todas as atividades. } \\
\text { Acredito que seja a dinâmica do trabalho o bombardeio de informaçôes." }\end{array}$ \\
\hline 4 & $\begin{array}{l}\text { "As crianças se envolvem bem, ficam atentas às estratégias, mas demonstram dificuldades em entender como } \\
\text { fazer a atividade."; "A sessão foi bem dinâmica. As crianças gostaram, respondendo a tudo que foi proposto, } \\
\text { responderam bem quanto às palavras iguais e diferentes, as letras e os sons, as trocas de letras para formar uma } \\
\text { nova palavra." }\end{array}$ \\
\hline 5 & $\begin{array}{l}\text { "Hoje as crianças estavam bem envolvidas, porém ainda continuo achando muita informaçáo para o tempo. As } \\
\text { estratégias são muito boas, as meninas conseguem passar o que elas querem em cada atividade, mas como para } \\
\text { a turma é um trabalho novo, elas necessitam de um tempo maior para assimilar as atividades propostas."; "Foi } \\
\text { bem legal, as crianças gostaram e conseguiram responder bem nas atividades de rima, palavras iguais e diferentes, } \\
\text { na atividade que retirava uma letra para formar nova palavra." }\end{array}$ \\
\hline 6 & $\begin{array}{l}\text { "O tempo para cada atividade é pouco e eles precisam de mais tempo para compreender melhor o que é solici- } \\
\text { tado e treinar mais em cada sessão. Acredito que se tivesse um apoio da escrita, facilitaria a compreensão."; "As } \\
\text { crianças não foram bem na sequência de palavras sem sentido. As estratégias dos terapeutas foram boas."; "As ati- } \\
\text { vidades são muito boas, apesar de rápidas, mas eles apresentam grande dificuldade de compreensão e memória, } \\
\text { além do vocabulário reduzido, o que dificulta o trabalho." }\end{array}$ \\
\hline 7 & $\begin{array}{l}\text { "As crianças se envolvem, mas falta mais explicação de cada atividade explorada. O tempo de cada uma é muito } \\
\text { curto. As crianças não entenderam a atividade de repetir as sílabas, a de rimas, mas, mesmo assim, já se passa } \\
\text { para outra. Sei que o trabalho é fonológico, mas tenho certeza que se, na explicaçáo, usasse a escrita, facilitaria."; } \\
\text { "Em todas as atividades foram apresentadas uma boa dinâmica. As crianças conseguiram se envolver bem nas } \\
\text { atividades, tiveram boa compreensão dos trabalhos apresentados e também devido a um recurso a mais que a } \\
\text { terapeuta usou na rima. Continuo achando pouco tempo."; "Brincadeiras muito boas e diversificadas. Ainda as } \\
\text { mesmas dificuldades. Tempo curto." }\end{array}$ \\
\hline 8 & $\begin{array}{l}\text { "Todos conseguiram saber o nome da letra, porém os sons das letras só conseguiram com ajuda da fono. Acre- } \\
\text { dito que com o tempo maior, ou seja, mais repetiçôes, eles conseguiriam."; "As estratégias para a realizaçấo das } \\
\text { atividades foram ótimas e bastante diversificadas. O tempo da atividade é muito pouco." }\end{array}$ \\
\hline 9 & $\begin{array}{l}\text { "As atividades elaboradas para as crianças foram bastante interessantes. Observei uma evolução de segunda-feira } \\
\text { para hoje no sentido de melhor compreensão e melhores respostas. Nos dois atendimentos observados a rima } \\
\text { foi o que mais dificuldade apresentaram. Quando foi solicitada a tarefa de tirar e botar sílabas nas palavras, } \\
\text { nenhuma das crianças conseguiu acertar."; "Todos se envolvem bem nas atividades, têm boas dinâmicas e ótimas } \\
\text { estratégias."; "Melhoraram no reconhecimento de letras e de alguns dos seus sons. A dificuldade de memoriza- } \\
\text { çâo, compreensão e vocabulário restrito, dificulta bastante a compreensão do trabalho." }\end{array}$ \\
\hline
\end{tabular}




\begin{tabular}{|c|c|}
\hline Sessão & Análise das Pedagogas \\
\hline 10 & $\begin{array}{l}\text { "Teve dúvidas na tarefa de rima, mas quando a terapeuta repetia e explicava novamente, ela acertava."; "Para } \\
\text { melhor compreensão do grupo sobre a realização da atividade, é preciso que seja chamada a atenção de todos } \\
\text { para as instruçóes e essa deve ser passada pausadamente, principalmente quanto aos jogos." }\end{array}$ \\
\hline 11 & $\begin{array}{l}\text { "A tarefa de dizer o nome da letra da família e depois o som, não conseguiu acertar. Apresentaram dificuldades } \\
\text { na tarefas de rima, palavras iguais e diferentes, tirar sílaba da palavra e acrescentar sílaba na palavra."; "Todos } \\
\text { compreenderam as instruçóes dada a cada atividade, as estratégias foram ótimas, o que facilitou o envolvimento } \\
\text { de todos."; "Gostei das estratégias para cada atividade, o que favoreceu o envolvimento do grupo, bem como a } \\
\text { melhor compreensão por parte da maioria dos participantes." }\end{array}$ \\
\hline 12 & $\begin{array}{l}\text { "O tempo para cada atividade foi muito bom. Diante da boa explicaçáo das atividades, todos tiveram bom } \\
\text { envolvimento e bom desempenho." }\end{array}$ \\
\hline 13 & $\begin{array}{l}\text { "Dificuldade na aliteração. Precisa reforçar na repetição dos números."; "As estratégias apresentadas nas ativi- } \\
\text { dades foram bem atrativas, foram bem orientadas."; "Boas estratégias em cada atividade, excelente interação da } \\
\text { terapeuta com o grupo e bons resultados." }\end{array}$ \\
\hline 14 & $\begin{array}{l}\text { "Dificuldade nas palavras que rimam, poucas crianças compreendem."; "Mesmo com boa explicação das } \\
\text { estratégias apresentadas, os dois estavam muito desatentos."; "Muito boa sessão."; "Bom envolvimento de todos } \\
\text { nas atividades, o tempo foi bom e as estratégias bem dinâmicas e bem aplicadas, o que facilita também no } \\
\text { desempenho e resultado." }\end{array}$ \\
\hline 15 & $\begin{array}{l}\text { "Muito boa a atividade do Twister "; "Eles apresentam muita dificuldade na compreensão, no vocabulário e na } \\
\text { memória, o que dificulta muito o trabalho."; "A estratégia para cada atividade foi bem legal, dinâmica e bem } \\
\text { explicada, o que favoreceu no desempenho e resultado da maioria das atividades." }\end{array}$ \\
\hline 16 & "Na atividade de encontrar a letra equivalente ao som, eles 'chutam', vão por 'ensaio e erro', por eliminação." \\
\hline 17 & "Essa turma é mais lenta nas respostas, precisando que as orientaçôes fossem repetidas mais de uma vez." \\
\hline 18 & “Todos apresentaram dificuldade na memorização." \\
\hline 19 & $\begin{array}{l}\text { "Todos participaram com bastante interação e tiveram boa compreensão do que foi orientado."; "Turma boa de } \\
\text { se trabalhar e que dáo bons resultados, apesar das faltas. Atendimento dinâmico e interativo, com boas estraté- } \\
\text { gias de trabalho muito bem explicadas." }\end{array}$ \\
\hline 20 & "Pelo som descobriram o nome da letra. Acertaram as sílabas iguais e palavras iguais ou diferentes." \\
\hline
\end{tabular}

Quadro 1. Observações das pedagogas participantes sobre cada uma das sessōes de intervenção. Fonte: elaboração própria

\section{Discussáo}

Nesta pesquisa, os resultados obtidos corroboram com os encontrados na literatura, de que há a necessidade de adaptaçôes para a intervenção fonológica para crianças com perda auditiva (Easterbrooks, Lederberg, \& Connor, 2010; Lederberg et al., 2014; Lee, Hall, \& Sancibrian, 2017).

O programa intervenção fonológica elaborado baseado nos modelos de remediação de Capellini et al. (2010), Salgado (2010) e na revisão da literatura sobre programas de intervenção fonológica para crianças com deficiência auditiva, em outras línguas (Lederberg et al., 2014; Lee, Hall, \& Sancibrian, 2017; Spencer et al., 2008), necessitou de ajustes específicos para a amostra estudada, o que reforça a necessidade da continuidade de pesquisas envolvendo o referido programa, aplicado a diferentes amostras, para a validação de sua aplicabilidade na população de crianças com deficiência auditiva. 
Segundo Lederberg et al. (2014), crianças com perda auditiva, em níveis pré-escolares, podem desenvolver a habilidade linguística de leitura a partir de instruçóes e de estratégias especificamente adaptadas às suas necessidades. O mesmo mostrou-se verdadeiro para as crianças em nível escolar mais avançado, participantes desta pesquisa, a partir da análise dos planos diários da intervenção.

Todas as sessões sofreram algum tipo de adaptação, seja de estratégia, instrução ou de material, tendo sido observado mais de um tipo de adaptação por sessão, especialmente nas primeiras sessões. Quanto ao tipo de adaptação, a literatura específica retrata, em especial, o uso de apoio visual (materiais escritos ou concretos), que facilite à criança com deficiência auditiva o acesso à tarefa fonológica (Chen, 2014; Easterbrooks, Lederberg, \& Connor, 2010; Gilliver et al., 2016; Lee, Hall, \& Sancibrian, 2017; Miller, Lederberg, \& Easterbrooks, 2013; Nakeva et al., 2013; Rakhshanfadaee \& Salehi, 2016; Wang et al., 2013; Werfel, Douglas, $\&$ Ackal, 2016), sendo este o segundo tipo de apoio mais observado nesta pesquisa. $\mathrm{O}$ apoio mais frequente aos participantes desta pesquisa foi o uso de destaques acústicos. Tais modificaçóes foram realizadas especialmente em virtude da dificuldade observada nas crianças em compreender e executar as atividades no tempo destinado à sessão, bem como ao seu nível de conhecimento prévio das habilidades fonológicas, observação que concorda com a literatura (Spencer et al., 2008).

A análise dos planos diários das sessões também permitiu elencar as principais estratégias de comunicação e técnicas auditivas utilizadas pelas pesquisadoras durante o trabalho fonológico. Ressalta-se que uma ampla gama de técnicas pode ser empregada de acordo com a necessidade e habilidades a serem trabalhadas, para o favorecimento do desempenho das crianças nesse tipo de treinamento, que extrapola a terapia fonoaudiológica convencional (Granha, Oliveira, \& Rumpf, 2008; Resegue-Coppi, 2008).

Observou-se que as características das crianças dos grupos de intervenção certamente determinaram as necessidades de adaptaçóes a serem realizadas (Lederberg, Schick, \& Spencer, 2013), com destaque para o tempo de privação sensorial (Bornstein, 2018; Wake \& Carew, 2016), o uso restrito dos aparelhos auditivos para algumas delas (Penna, Lemos, \& Alves, 2015; Vasconcelos \& Pereira, 2015) e o ingresso tardio no programa de (re)habilitação auditiva (Bicas, Guijo, Delgado-Pinheiro, 2017; Oliveira, Penna, \& Lemos, 2015; Tabaquim et al., 2013), os quais foram fatores verificados na amostra e que devem ser considerados na elaboração das estratégias para o trabalho fonológico.

Ainda assim, todas as crianças deste estudo demonstraram avanços em suas habilidades, o que pôde ser observado na análise dos resultados das sessóes, fato que também corrobora as pesquisas sobre a intervenção fonológica para crianças com deficiência auditiva com maior ou menor acesso aos sons (Bergeron et al., 2009; Gamba-Yoshida, 2017; Goldberg \& Lederberg, 2015; Lederberg, Schick, \& Spencer, 2013), destacando a primazia das habilidades fonológicas para a aquisição do código escrito, tão fundamental para o fortalecimento do desenvolvimento da linguagem e da cognição, entre outras habilidades na criança com deficiência auditiva (Harris, Terlektsi, \& Kyle, 2017; Lemes \& Goldfeld, 2008; Mahapatra, 2015).

As habilidades que tiveram um índice mínimo de adaptações, desde o início do programa diminuindo com o passar dos dias foram: correspondência grafema-fonema e fonema- 
-grafema, de acordo com as análises dos planos diários e das fichas de observação, resultado comparado ao estudo de Nakeva von Mentzer et al. (2013), no qual a melhora na relação fonema-grafema foi considerada de moderada a forte, bem como ao estudo de Werfel, Douglas, \& Ackal (2016), que relata o ganho das crianças no início do programa das habilidades de relação grafema-fonema, para posteriormente observar melhora na habilidade de rimar.

Destaca-se ainda que são escassas as pesquisas que relatam a percepção de profissionais da educação em relação ao trabalho de intervenção fonológica para crianças com deficiência auditiva. Notou-se que a percepção das pedagogas participantes deste estudo se deteve nas dificuldades das crianças, sem a utilização dos termos técnicos para a sua definição, o que dirige a atenção para a necessidade de formação específica dos pedagogos envolvidos em programas de (re)habilitação de crianças com deficiência auditiva (Bevilacqua \& Formigoni, 2012; Moret, 2017) para o trabalho com as habilidades fonológicas, conforme referido por Messier e Jackson (2014) e Spencer et al. (2008), que alertam sobre a necessidade da proficiência em consciência fonológica de educadores que trabalham com crianças com deficiência auditiva.

\section{Conclusão}

Conclui-se que o programa de intervenção fonológica para crianças com deficiência auditiva necessitou de adaptações referentes às instruçóes oferecidas aos grupos, às estratégias propriamente ditas, bem como aos materiais utilizados, destacando-se a importância dos registros detalhados dos resultados das sessóes de programas dessa natureza, dada a importância da análise das estratégias para a elaboração de intervenções cada vez mais efetivas e eficazes para essa população.

A partir das observações realizadas para as crianças desta pesquisa recomenda-se a continuidade dos estudos que se proponham validar programas de intervençáo voltados ao desenvolvimento da leitura para crianças com deficiência auditiva e que os serviços de (re)habilitação auditiva considerem, em seu planejamento, a instrução específica com as habilidades fonológicas, dada a sua relevância para a aquisição e o desenvolvimento da leitura e da escrita, agregando à criança o domínio de outras habilidades linguísticas e cognitivas, importantes para sua plena inclusão. Além disso, destaca-se a preocupação em treinar os profissionais que desenvolvem este trabalho com as crianças, especialmente no enquadre de grupo.

As principais limitaçóes deste estudo referiram-se às características da amostra tais como: número de sujeitos, a faixa etária dos grupos trabalhados, bem como a sua aplicação em um único serviço de (re)habilitação auditiva, com necessidade de ampliação do número amostral, da estratificação da análise das estratégias da intervenção fonológica aplicada a crianças de diferentes faixas etárias, bem como a avaliação da implementação dessas intervençóes por profissionais da pedagogia especializada em programas de (re)habilitação auditiva.

Destaca-se o ineditismo desse tipo de estudo e identifica-se como potencial da referida pesquisa a construção e a análise pormenorizada do material da intervenção aplicada, com a possibilidade de tornar-se um referencial para o desenvolvimento de programas de treinamento de habilidades fonológicas em enquadre terapêutico grupal, específico para a população de crianças com deficiência auditiva. 


\section{REFERÊNCIAS}

Bergeron, J. P., Lederberg, A. R., Easterbrooks, S. R., Miller, E. M., \& Connor, C. M. (2009). Building the alphabetic principle in young children who are deaf and hard of hearing. The Volta Review, 109(2-3), 87-119.

Bevilacqua, M. C., \& Formigoni, G. M. P. (2012). Audiologia educacional: uma opção terapêutica para a criança deficiente auditiva. São Paulo: Pró-Fono.

Bicas R. S., Guijo L. M., \& Delgado-Pinheiro E. M. C. (2017). Oral communication and auditory skills of hearing-impaired children and adolescents and the speech therapy rehabilitation process. Rev. CEFAC, 19, 465-474. DOI: http://dx.doi.org/10.1590/1982-0216201719412516

Bornstein, S. (2018). The importance of early identification and intervention for children with hearing loss. Part 1: Human Development. J Health Commun, 3(8). DOI: 10.4172/2472-1654.100119

Capellini, S. A., Sampaio, M. N., Kawata, K. H. S., Padula, N. A M. R., Santos, L. C. A., Lorencetti, M. D., \& Smythe, I. (2010). Eficácia terapêutica do programa de remediação fonológica em escolares com dislexia do desenvolvimento. Biofisica, 12(6), 27-39. DOI: http://dx.doi.org/10.1590/S151618462009005000060

Chen, Y. H. (2014). Effects of phonological awareness training on early chinese reading of children who are deaf and hard of hearing. Volta Review, 114(1), 85-100.

Easterbrooks, S. R., Lederberg, A. R., \& Connor, C. M. (2010). Contributions of the emergent literacy environment to literacy outcomes for young children who are deaf. American Annals of the Deaf, 155(4), 467-480. DOI: 10.1353/aad.2010.0024

Gabriel, R., Morais, J., \& Kolinsky, R. (2016). A aprendizagem da leitura e suas implicaçóes sobre a memória e a cognição. Ilha do Desterro, 69(1), 61-78. DOI: http://dx.doi.org/10.5007/21758026.2016v69n1p61

Gamba-Yoshida, M. C. C. (2017). Atividades de estimulaçâo da consciência fonológica por meio de um livreto destinado a alunos com deficiência auditivalsurdez (Dissertaçáo de Mestrado). Universidade Estadual Paulista Júlio de Mesquita Filho - Faculdade de Ciências, Bauru, São Paulo, SP, Brasil.

Gilliver, M., Cupples, L., Ching, T. Y. C., Leigh, G., \& Gunnourie, M. (2016). Developing sound skills for reading: teaching phonological awareness to preschoolers with hearing loss. Journal of Deaf Studies and Deaf Education, 21(3), 268-279. DOI: https://doi.org/10.1093/deafed/enw004

Goldberg, H. R., \& Lederberg, A. R. (2015). Acquisition of the alphabetic principle in deaf and hardof-hearing preschoolers: the role of phonology in letter-sound learning. Reading and Writing, 28(4), 509-525. DOI: https://doi.org/10.1007/s11145-014-9535-y

Granha, D. O., Oliveira, A. S., \& Rumpf, K. (2008). Estratégias terapêuticas realizadas com crianças deficientes auditivas oralizadas: revisão de literatura. In P. B. Silva, \& R. H. F. Dav (Eds.), Cadernos da Fonoaudiálogo. Audiologia (pp. 49-55). São Paulo: Lovise.

Harris, M., Terlektsi, E., \& Kyle, F. E. (2017). Concurrent and longitudinal predictors of reading for deaf and hearing children in Primary School. The Journal of Deaf Studies and Deaf Education, 22(2), 233-242. DOI: 10.1093/deafed/enw101

Lederberg, A. M., Miller E. M., Easterbrooks S., \& Connor, C. M. (2014). Foundations for literacy: an early literacy intervention for deaf and hard-of-hearing children. Journal of Deaf Studies and Deaf Education, 19(4), 438-455. DOI: https://doi.org/10.1093/deafed/enu022 
Lederberg, A. R., Schick, B., \& Spencer, P. E. (2013). Language and literacy development of deaf and hard-of-hearing children: Successes and challenges. Developmental Psychology, 49(1), 15-30. DOI: https://doi.org/10.1037/a0029558

Lee, S. A. S., Hall, B., \& Sancibrian, S. (2017). Feasibility of a supplemental phonological awareness intervention via telepractice for children with hearing loss: a preliminary study. International Journal of Telerehabilitation, 9(1), 23-38. DOI: https://doi.org/10.5195/ijt.2017.6216

Lemes, J. P., \& Goldfeld, M. (2008). Analysis of orthographic errors of children with cochlear implants. Rev Soc Bras Fonoaudiol., 13(3), 179-189.

Mahapatra, S. (2015). Improving reading outcomes for students with or at risk for reading disabilities: a synthesis of the contributions from the Institute of Education Sciences Research Centers. Journal of Education and Practice, 6(19), 57-63.

Messier, J., \& Jackson, C. W. (2014). A comparison of phonemic and phonological awareness in educators working with children who are deaf or hard of hearing. American Annals of the Deaf, 158(5), 522-538.

Miller, E. M., Lederberg, A. R., \& Easterbrooks, S. R. (2013). Phonological awareness: explicit instruction for young deaf and hard-of-hearing children. Journal of Deaf Studies and Deaf Education, 18(2), 206-227. DOI: https://doi.org/10.1093/deafed/ens067

Moret, A. L. M. (2017). Audiologia Educacional na Infância: contribuiçóes do Campus de Bauru da Universidade de São Paulo. In S. Silva, \& L. Digiampietri (Orgs.), Conhecendo a USP - contribuiçóes do ensino, da pesquisa e da extensão no campo das deficiências (pp. 33-48). São Paulo: FEUSP.

Nakeva von Mentzer, C., Lyxell, B., Sahlén, B., Wass, M., Lindgren, M., Ors, M., Kallioinen, P., \& Uhlén, I. (2013). Computer-assisted training of phoneme-grapheme correspondence for children who are deaf and hard of hearing: Effects on phonological processing skills. International Journal of Pediatric Otorhinolaryngology, 77(12), 2049-2057. DOI: https://doi.org/10.1016/j.ijporl.2013.10.007

Oliveira, P. S, Penna, L. M., \& Lemos, S. M. A. (2015). Language development and hearing impairment: literature review. Rev. CEFAC, 17, 2044-2055. DOI: http://dx.doi.org/10.1590/19820216201517611214

Penna, L. M., Lemos, S. M. A., \& Alves, C. R. L. (2015). Auditory and language skills of children using hearing aids. Braz J Otorhinolaryngol, 81, 148-157. DOI: http://dx.doi.org/10.1016/j. bjorl.2014.05.034

Rakhshanfadaee, A., \& Salehi, M. (2016). phonological awareness in children with hearing loss. The Hearing Journal, 69(9), 32-35.

Resegue-Coppi, M. M. (2008). Desenvolvendo as habilidades auditivas em crianças usuárias de implante coclear: estratégias terapêuticas (Dissertação de Mestrado em Fonoaudiologia). Faculdade de Odontologia de Bauru, Universidade de São Paulo, Bauru, São Paulo, Brasil.

Salgado, C. A. (2010). Programa de remediação fonológica, de leitura e escrita em crianças com dislexia do desenvolvimento (Tese de doutorado). Faculdade de Ciências Médicas da Universidade Estadual de Campinas, São Paulo, São Paulo, Brasil.

Spencer, E. J., Schuele, C.M., Guillot, K. M. \& Lee, M.W. (2008). Phonemic awareness skill of speechlanguage pathologists and other educators. Language, Speech, and Hearing Services in Schools, 39, 512-520. 
Tabaquim, M. de L. M., Nardi, C. G. A., Ferrari, J. B., Moretti, C. N., Yamada, M. O., \& Bevilacqua, M. C. (2013). Avaliação do desenvolvimento cognitivo e afetivo-social de crianças com perda auditiva. Revista CEFAC, 15(6), 1475-1481. DOI: http://dx.doi.org/10.1590/S1516-18462013005000051

Trad, L. A. B. (2009). Grupos focais: Conceitos, procedimentos e reflexóes baseadas em experiências com o uso da técnica em pesquisas de saúde. Physis, 19(3), 777-796. DOI: https://doi.org/10.1590/ s0103-73312009000300013

Vasconcelos, K. A. de, \& Pereira, M. D. G. A. (2015). Importância da educação formal dos responsáveis e renda familiar no processo de adesão ao uso de próteses auditivas em menores de 12 anos. Revista CEFAC, 17(1), 216-222. DOI: http://dx.doi.org/10.1590/1982-0216201519213

Wake, M., \& Carew, P. (2016). Science, not philosophy, will help deaf and hard-of-hearing children reach their potential. Pediatrics, 137(1), 1-2. DOI: https://doi.org/10.1542/peds.2015-3443

Wang, Y., Spychala, H., Harris, R. S., \& Oetting, T. L. (2013). The effectiveness of a phonics-based early intervention for deaf and hard of hearing Preschool children and its possible impact on reading skills in Elementary School: a case study. American Annals of the Deaf, 58(2),107-120.

Werfel, K. L., Douglas, M., \& Ackal, L. (2016). Small-Group phonological awareness training for Pre-Kindergarten children with hearing loss who wear Cochlear Implants and/or Hearing Aids. Deafness and Education International, 18(3), 134-140. DOI: https://doi.org/10.1080/14643154. 2016.1190117-

Recebido em: 08/02/2019

Reformulado em: 13/11/2019

Aprovado em: 02/01/2020 
MARINHO, A.L.C.; ROCHA E SILVA, B.L.; BRAZOROTTO, J.S. 


\section{Errata}

No artigo "Análise das Estratégias Empregadas em um Programa de Intervenção Fonológica para Crianças com Deficiência Auditiva"

"Analysis of Strategies Employed in a Phonological Intervention Program for Hard of Hearing Children"

Onde se lê: http://dx.doi.org/10.1590/s1413-65382620000100002

Leia-se: https://doi.org/10.1590/1980-54702020v26e0018 